\title{
Le harcèlement moral au travail dans la législation espagnole
}

Du risque psychosocial à l'obligation de sécurité de l'employeur

Moral harassing at work in the Spanish law of Prevention of Occupational

hazards and the obligation of safety for employers

\section{Carmen Grau Pineda}

\section{OpenEdition}

\section{Journals}

Édition électronique

URL : http://journals.openedition.org/travailemploi/2187

DOI : $10.4000 /$ travailemploi.2187

ISSN : 1775-416X

\section{Éditeur}

DARES - Ministère du Travail

\section{Édition imprimée}

Date de publication : 30 décembre 2007

Pagination : 49-61

ISSN : 0224-4365

\section{Référence électronique}

Carmen Grau Pineda, «Le harcèlement moral au travail dans la législation espagnole », Travail et Emploi [En ligne], 112 | octobre-décembre 2007, mis en ligne le 05 novembre 2010, consulté le 01 mai 2019. URL : http://journals.openedition.org/travailemploi/2187 ; DOI : 10.4000/travailemploi.2187 


\title{
Le harcèlement moral au travail dans la législation espagnole: du risque psychosocial à l'obligation de sécurité de l'employeur
}

\author{
Carmen Grau Pineda (*)
}

L'article aborde la question du harcèlement moral au travail dans la perspective juridique des politiques de prévention des risques au travail qui s'imposent à l'employeur. Il s'appuie sur un panorama des dispositions législatives actuellement en vigueur en Espagne et de la jurisprudence des tribunaux espagnols, mais aussi de documents institutionnels communautaires et internationaux. Il met en évidence la difficulté de construction du concept de harcèlement moral au travail, que l'auteure distingue des phénomènes proches comme le mobbing, le stress ou le harcèlement discriminatoire, et montre l'incertitude qui existe encore dans la législation espagnole autour de la définition du phénomène, malgré une ouvre jurisprudentielle considérable. L'auteure souligne l'impact important que devraient avoir les normes communautaires dans ce domaine, faisant ressortir la nécessité de mesures de prévention pour la mise en place d'une protection efficace dans les cas de harcèlement au travail.

Pour mieux comprendrel'apparition des nouveaux risques professionnels que sont les risques psychosociaux, il faut faire un retour rapide sur le mouvement de déqualification du travail qui a accompagné l'industrialisation de la production et l'avènement du système taylorien, caractérisé par le travail à la chaîne, la répétition des tâches et la rapidité d'exécution en vue d'une plus grande production. Parmi les conséquences les plus évidentes de cette transition caractérisée par la primauté absolue des aspects économiques sur le reste de facteurs, en particulier sociaux, on observe le durcissement des charges et des conditions de travail, ainsi que la multiplication des contrôles et de la surveillance sur les employés, associés à l'effondrement de la perception psychologique et sociale du travail comme activité créative et activité de production utile(1). Mais, si la préoccupation pour les risques physiques et psychiques est apparue dans la transition vers la société industrielle, la prise en considération des risques psychosociaux semble propre aux sociétés postindustrielles

(*) Professeur à l'université de Las Palmas de Gran Canaria (ULPGC), cgrau@ddp.ulpgc.es Ce travail, complété et développé, a son origine dans une communication présentée au XVIII ${ }^{e}$ Congrès mondial de droit du travail et de la Sécurité sociale, Paris, 5-8 septembre 2006, préparée pendant un séjour de recherche dans le Centre de droit comparé du travail et de la sécurité sociale (COMPTRASEC UMR CNRS 5114) de l'université Montesquieu-Bordeaux IV. L'auteure remercie Philippe Martin, chargé de recherche CNRS (COMPTRASEC) pour son aide à la traduction du présent article.

(1) En ce sens, $c f$. la lecture très intéressante du travail de BONNECHĖRE, 1994, pp. 173 et suivantes.
(Molina NaVArRete, 2001). En effet, la globalisation économique et sociale, la tertiarisation de l'économie, la décentralisation ou l'externalisation des activités, les nouvelles technologies, la diversification de la force de travail et de la durée du travail, entre autres, ont et auront une incidence décisive sur le contenu des relations de travail et sur le modèle d'organisation du travail(2). Les nouvelles modalités de travail, incluant celles dérivées de la technologie de l'information, les changements dans l'organisation du travail et dans la durée du travail, la mise en œuvre de conduites individualisées orientées vers le rendement, «ont créé des bouleversements psychosomatiques prenant la forme de stress, de dépression ou d'anxiété, sans parler des problèmes posés par la violence dans le travail ou par le harcèlement» ( $c f$. RodríGuez PIÑERo, 2005, pp. 3 et suiv.; DASSA, 2002, pp. 255 et suiv.). Comme l'a souligné Serrano Olivares (2005), «toutes ces conditions productives et organisationnelles agiront dans une plus ou moins grande mesure comme des facteurs stressants et auront, par conséquent, une plus ou moins grande incidence sur la santé des travailleurs; cela dépend du niveau de soutien

(2) Selon le Conseil économique et social, Rapport sur les nouveaux risques pour la santé des salariés, 2004, CES, voir le site Internet www.ces.fr/ Taylor lui-même a souligné les «signes d'énervement» liés à certains postes tenus par des femmes dans les usines de fabrication de bicyclettes; après la crise des années 1970 qui a modifié les rapports entre entreprises s'est mis en place un nouveau système productif qui diffère du fordisme ayant dominé l'après-guerre, dans lequel certains risques - les risques psychosociaux - ont une incidence particulière. 
social (de la part des supérieurs ou des collègues de travail) et de compensations (estime, stabilité de travail, perspectives de promotion professionnelle, etc. ) dont bénéficient les travailleurs » (SERRANO Olivares, 2005, p. 109).

C'est dans ce contexte que le droit du travail développe des notions nouvelles comme celles de risque psychosocial, obligeant à reconsidérer le concept traditionnel de conditions de travail: en effet, ces notions, bien qu'elles fassent référence à des comportements ou à des pratiques connus, n'ont commencé à susciter une attention juridique que dans les dernières années.

Ces nouveaux risques, loin de représenter une question mineure ou de faible importance, constituent l'un des problèmes les plus graves de la santé au travail dans tous les pays, développés et moins développés. Selon des données officielles, ils représentent le second problème de santé au travail (après les problèmes ergonomiques) en Europe. En fait, selon la quatrième enquête européenne sur les conditions de travail menée par la Fondation européenne pour l'amélioration des conditions de vie et de travail (2007) (3), un travailleur sur vingt $(5 \%)$ déclare avoir été exposé à des brimades et/ou du harcèlement au cours des douze derniers mois. D'autres, dans la même proportion, affirment avoir été personnellement victimes de violence, moins du fait de collègues $(2 \%)$ que de personnes extérieures au lieu de travail (4\%). $28 \%$ des personnes au travail déclarent souffrir du stress, ce qui en fait le deuxième problème de santé invoqué après les maux de dos $(33 \%)$ et avant les douleurs musculaires du dos et des épaules $(23 \%)$ et la fatigue générale. Ceci explique que ces risques aient été placés au centre de l'attention de l'Union européenne, ainsi qu'en atteste la dernière Stratégie communautaire en matière de sécurité et de santé pour la période 2007-2012(4). La Stratégie fait de la promotion de la santé mentale au travail une priorité, et s'est fixée comme objectif non seulement de réduire le nombre des accidents et des maladies professionnelles, mais aussi de promouvoir un véritable «bien-être au travail», tant au plan physique que moral ou social, et qui ne se mesure pas seulement à l'absence d'accidents ou de maladies professionnelles. Après avoir souligné que «[...] les mutations dans l'organisation du travail (obligation de résultats et plus grande flexi-

(3) Selon les enquêtes de la Fondation européenne pour l'amélioration des conditions de vie et de travail disponibles sur le site www.eurofound.europa.eu/publications

(4) Communication de la Commission des Communautés européennes du 21 février 2007 «Améliorer la qualité et la productivité du travail: une stratégie communautaire pour la santé et la sécurité au travail (2007-2012), sur le site eurlex.europa.eu. $C f$. aussi «La Estrategia Comunitaria de Salud y Seguridad en el Trabajo (2007-2012) Seguridad y Salud en el Trabajo », revista del INSHT, n' 42, 2007, pp. 36-44, sur le site www.mtas.es/insht/revista/index.htm bilité) ont une incidence profonde sur la santé au travail et plus généralement sur le bien-être des travailleurs [...]», la communication de la commission insiste sur le fait que " [...] on observe que certaines maladies comme le stress, la dépression, la violence, le harcèlement et l'intimidation dans le travail augmentent fortement et représentaient déjà, en $1999,18 \%$ des problèmes de santé en rapport avec le travail [...]». Pour accomplir cet objectif de bien-être au travail, des mesures complémentaires s'imposent, parmi lesquelles figure en bonne place la prévention des risques psychosociaux.

En outre, il est important d'indiquer que les partenaires sociaux européens (CES, BUSINESSEUROPE, CEEP et UEAPME) se sont engagés à lutter contre la violence et le harcèlement au travail en signant le 26 avril 2007 à Bruxelles un accord-cadre sur le harcèlement et la violence au travail. Cet accord vise à prévenir et gérer des situations de brimade, harcèlement sexuel et violence physique survenant sur le lieu de travail. Les entreprises établies en Europe auront ainsi l'obligation d'adopter une politique de tolérance zéro à l'égard de tels comportements et de mettre en place des procédures de règlement d'éventuels cas de harcèlement et de violence. L'accord européen condamne toute forme de harcèlement et de violence et renvoie à l'obligation qu'ont les employeurs de protéger les salariés contre de telles situations. Les entreprises devront mettre en place des procédures de gestion des cas de harcèlement ou de violence. Ces procédures pourront comprendre une étape informelle consistant dans l'intervention d'une personne qui aura la confiance de la direction et des travailleurs. Les plaintes devront être traitées rapidement et donner lieu à une enquête. Les principes de dignité, de confidentialité, d'impartialité et d'équité devront être respectés. L'accord souligne que des mesures appropriées seront prises à l'égard du contrevenant, notamment action disciplinaire, voire licenciement, tandis que la victime recevra une aide à la réintégration si nécessaire (5).

En Espagne, la tentative de spécifier ce phénomène a donné lieu à plusieurs documents de travail et rapports, parmi lesquels on mentionnera les rapports CISNEROS (2001-2002) et RANDSTAD (2003), qui avancent des chiffres absolument non concordants(6), permettant ainsi de constater la méconnaissance généralisée du phénomène qu'ils prétendent mesurer. Il en ressort principalement la difficulté à définir ce qu'est le harcèlement moral au travail et la difficulté à le distinguer de phénomènes voisins. L'enquête européenne sur les condi-

(5) $C f$. le site ec.europa.eu/employment_social/ Framework agreement on harassment and violence at work.

(6) Alors que le rapport Cisneros révèle que $16 \%$ de la population salariée souffre de harcèlement psychologique au travail, le rapport Randstad place le chiffre autour de $3 \%$ de la population occupée. 
tions de travail de 2007 montre que de nombreux travailleurs sont touchés par des phénomènes liés à la charge mentale de travail: nécessité de maintenir une attention importante pendant plus de la moitié de la journée de travail $(54,7 \%)$, rythmes de travail excessifs $(40,1 \%)$, réalisation de tâches répétitives et de courte durée $(39,4 \%)$. Les femmes, les travailleurs de moins de 45 ans et les salariés d'entreprises de taille importante sont soumis plus fréquemment à des situations de harcèlement psychologique au travail(7).

Dans ce contexte, le présent travail, à caractère juridique, ne se focalisera pas tant sur la question de la réparation du harcèlement moral que sur sa dimension préventive; l'article part de la prémisse qu'il existe une connexion directe entre harcèlement moral et organisation du travail, qui justifie la nécessité d'intégrer les facteurs de risque psychosocial dans les processus d'évaluation des risques au travail et de planification de l'activité préventive. Au préalable, il est nécessaire d'introduire quelques précisions terminologiques et de présenter le cadre normatif relatif au harcèlement moral au travail.

\section{Des précisions terminologiques préalables}

Il est fait ici référence à des concepts - ceux de risques psychosociaux, harcèlement moral, mobbing, stress, etc. - qui, bien qu'ils puissent paraître familiers dans des domaines étrangers à la sphère juridique (médecine, psychologie, etc.) sont généralement source de confusion dès lors qu'il s'agit d'en déterminer en droit la signification précise et la portée particulière.

Les risques psychosociaux ont été définis par l'OIT (1986) comme «les interactions entre le contenu, l'organisation et la gestion du travail et les conditions environnementales, d'une part, et les fonctions et les obligations des travailleurs, d'autre part. Ces interactions pourraient exercer une influence nuisible sur la santé des travailleurs à travers leurs perceptions et leur expérience». Les facteurs de risque psychosocial peuvent être multiples: individuels (ils peuvent affecter un travailleur en particulier), liés au contenu du travail (excès de travail, travail monotone), aux tâches individuelles ou aux caractéristiques personnelles, ils peuvent dériver du contexte d'organisation de l'entreprise (modèle d'organisation autoritaire et non transparent, rôle du salarié dans l'organisation, incertitudes sur le développement de la carrière professionnelle,

(7) Plus de détails sur l'enquête dans «Encuesta de Condiciones de Trabajo $2005 »$, Seguridad y Salud en el trabajo», Revista del INSHT, no 41, 2007, pp. 6 y ss, sur le site www.mtas.es/ insht/revista/index.htm. manque de reconnaissance, manque de ressources humaines et matérielles pour effectuer les tâches confiées, changements dans l'organisation), ou bien il peut s'agir de facteurs externes à l'entreprise (problèmes dans les relations interpersonnelles avec des clients, conflits familiaux).

Parmi la vaste gamme des risques psychosociaux, celui qui est au centre de la présente étude est le harcèlement moral au travail. Il faut au demeurant tenir compte de ce qu'aucune situation dans les entreprises n'est exempte de problèmes, d'évolutions et de changements et que les mauvais fonctionnements dans l'organisation font partie de la dynamique actuelle dans laquelle évoluent les entreprises. Ceci a pour conséquence que, dans la pratique, on associe stress, violence, souffrance et harcèlement moral, en plaçant ces notions sur un plan d'égalité et sans différentiation (LAPEYRIÈRE, 2004). Toutefois, il existe différentes classifications des risques psychosociaux, élaborées dans le champ de la psychologie, notamment organisationnelle et clinique, parmi lesquelles l'article prête une attention particulière au harcèlement moral au travail.

L'incidence des nouveaux risques psychosociaux est cependant loin d'être homogène. Selon les données officielles, $28 \%$ des travailleurs européens (41 millions) estiment subir un stress comme conséquence directe de leur travail (AGENCE EUROPÉENNE POUR LA SÉCURITÉ et la SANTÉ DANS le traVAIL, 2002 ) et environ $5 \%$ indiquent avoir été victimes de violence au travail ou de harcèlement moral (FondATION EUROPÉENNE POUR L'AMÉLIORATION DES CONDITIONS DE VIE ET DE TRAVAIL, 2007).

À titre préalable, il convient de préciser que toutes les situations de souffrance psychique ne conduisent pas à des situations de mobbing. Il faut faire attention à la généralisation de l'utilisation du terme mobbing. Alors que le terme mobbing suppose une attaque et qu'il est en rapport avec la réaction qui se produit dans le sujet qui en est la victime (c'est une modalité spécifique de stress au travail), le terme de harcèlement est plus vaste, et renvoie au comportement de celui qui en est l'auteur et au comportement négatif de ce dernier, indépendamment de la réaction de la victime. Cependant, dans la majorité des cas, on trouve des situations où, d'une manière ou d'un autre, les risques sont dissimulés car il y a en réalité une constellation de problèmes dont les limites de l'un à l'autre sont diffuses.

Toutefois, et comme on le verra dans les lignes qui suivent, les situations de harcèlement moral au travail recouvrent une addition de caractéristiques qu'il est très utile de distinguer pour différencier le harcèlement moral des autres risques psychosociaux.

En 1998, M.-F. Hirigoyen a publié ses observations sur « des patients qui se sentaient détruits par 
quelqu'un », en décrivant les aspects psychopathologiques des relations de harcèlement à la fois dans leur origine et dans leurs conséquences (HIRIGOYEN, 1998 , pp. 55 et suiv., 2004). Selon elle, il s'agit de l'exercice «d'une violence psychologique externe de la part d'une ou plusieurs personnes sur une autre dans le cadre du travail, par laquelle se crée une relation asymétrique de pouvoir, de manière systématique et prolongée dans le temps », considérée comme « une forme caractéristique de stress au travail, qui présente la particularité qu'elle ne se produit pas exclusivement en raison de causes directement en rapport avec l'exercice du travail ou avec son organisation, mais qui a son origine dans les relations interpersonnelles qui sont établies dans toute entreprise entre les différents individus $»$.

Une autre définition à laquelle nous souhaitons nous référer apparaît dans la note technique préventive (NTP) 476 de l'Institut national de sécurité et d'hygiène au travail espagnol (INSHT) $(8)$, selon laquelle il s'agit d'une «situation où une personne (ou, à de rares occasions, un groupe de personnes) exerce une violence psychologique extrême, de manière systématique et récurrente (en moyenne une fois par semaine) et pendant un temps prolongé (en moyenne environ six mois) sur une autre personne ou des personnes sur le lieu de travail, dans le but de détruire les réseaux de communication de la victime ou des victimes, de détruire sa réputation, de perturber l'exercice de ses tâches et d'obtenir, finalement, que cette personne ou ces personnes finissent en abandonnant le lieu de travail». En dérivent les caractéristiques objectives (systématicité et réitération) et subjectives (intentionnalité et but concret) du harcèlement moral dans le travail que nous approfondirons par la suite.

La jurisprudence espagnole a également fourni plusieurs définitions du harcèlement moral au travail, certaines d'entre elles étant très complètes, en insistant sur l'idée que ce type de harcèlement va au-delà d'un exercice inadéquat ou abusif du pouvoir de direction. Comme il a été spécifié par la décision du tribunal du travail de Madrid, en date du 18 juin 2001 (AS 2001\1667), il ne suffit pas que se produise un exercice arbitraire du pouvoir de direction ou bien que l'entreprise connaisse une situation conflictuelle et que le travailleur souffre, en conséquence, d'une situation de stress, pour être face à un cas de harcèlement moral, car ce qui caractérise cette dernière situation, c'est le recours à de nombreuses vexations dans une période prolongée, avec l'intention d'affecter de manière négative la personnalité de la victime. Dans le même sens, la décision du tribunal du travail de Murcie du 30 mai 2003 (confirmée par

(8) Disponible sur le site internet.mtas.es/Insht/ntp/ntp 476. htm
STSJ Murcie, 3 septembre 2003) signale qu' «il convient de distinguer de manière adéquate les conduites qualifiables de harcèlement des exactions qui peuvent être commises par l'employeur qui exerce de manière abusive ses pouvoirs de direction et d'organisation de l'entreprise. Il ne s'avère donc pas possible d'arriver à la conclusion que tout exercice abusif de ces pouvoirs peut être qualifié de harcèlement et cela, évidemment, sans préjudice des sanctions que prévoit la loi contre ces actions illégales ». Plus récemment, le TSJ de Madrid (décision du 5 octobre 2005(9)) signalait les limites du recours aux pouvoirs patronaux pour garantir dans toutes les situations une protection efficace en matière de sécurité et de santé au travail des travailleurs quand ils sont affectés par l'exercice de ces pouvoirs; il en résulte que cet exercice peut être considéré comme «irrégulier» lorsqu'il donne naissance à un certain type de risque professionnel pour la santé du travailleur et, par conséquent, suppose que l'employeur n'a pas satisfait à son devoir de sécurité et de santé au travail, par rapport aux travailleurs exposés aux risques professionnels dérivés d'un tel exercice.

On peut considérer que l'œuvre jurisprudentielle en Espagne a été considérable, dans la mesure où les tribunaux ont effectué une authentique tâche de construction de la signification juridique du terme de harcèlement moral à partir du concept clinique(10). En même temps, les tribunaux espagnols ont répondu dans leurs motivations aux demandes posées par les victimes de ce type de harcèlement, dans certains cas pour permettre la résiliation des contrats de travail(11), dans d'autres pour décider de la cessation immédiate des comportements préjudiciables et le paiement d'indemnités pour les dommages et les préjudices causés; ou pour pouvoir considérer les situations d'incapacité temporaire ou permanente associées à des comportements de harcèlement comme des accidents du

(9) Commentée par Martín Hernández, 2005.

(10) Entre autres, STS (contencioso-administrativo) de 23 de julio de 2001 (RJ 8027), STSJ País Vasco de 5 de julio de 2004 (JUR 202473), STSJ Castilla y León (Burgos) de 22 de abril de 2003 (AS 518), SJS no 7 de Murcia de 7 de marzo de 2003 (AS 1137), STSJ Cataluña de 25 de febrero de 2003 (JUR 117039), STSJ Galicia de 12 de septiembre de 2002 (AS 2603), STSJ Comunidad Valenciana (contencioso-administrativo) de 25 de septiembre de 2001 (RJCA 2002/548), STSJ Navarra de 15 de junio de 2001 (AS 230916), SJS n ${ }^{\circ} 2$ de Girona de 17 de septiembre de 2002 (AS 2691), SJS no 32 Barcelona de 16 de septiembre de 2002 (AS 2637), SJS no 1 Bilbao de 22 de marzo de 2002 (AS 2633), SJS no 2 Pamplona de 24 de septiembre de 2001 (AS 3192), SJS no 33 Madrid de junio de 2001 (AS 1667).

(11) SJS Pamplona de 24 de septiembre de 2001 (AS 3192), STSJ Cataluña de 28 de noviembre de 2001 (AS 249) y SJS Vigo de 28 de febrero de 2002 (AS 634). 
travail(12) méritant la protection spécifique que la Sécurité sociale espagnole prévoit pour les risques professionnels (13).

On a tenté, sans grand succès, de résoudre cette situation d'anomie, avec l'adoption de quelques initiatives législatives, dont la dernière est l'approbation de la loi 3/2007, sur l'égalité effectives entre les femmes et les hommes, comme on verra quelques lignes plus bas (GArcía-Perrote Escarpín, Mercader Uguina, 2007)(14). Mais il s'agit là de la standardisation d'un type bien précis de harcèlement, le harcèlement discriminatoire, lié à des raisons très concrètes (l'origine raciale ou ethnique, l'invalidité, l'orientation sexuelle) et non du harcèlement moral en particulier. Dans la législation espagnole, le harcèlement moral n'est pas considéré directement comme une pratique interdite. Le groupe parlementaire socialiste a présenté une proposition de loi sur le harcèlement moral au travail(15), mais sans aucun résultat jusqu'à présent.

En somme, et bien qu'il n'existe pas une unanimité totale dans la doctrine scientifique et judiciaire quant à la définition du harcèlement moral, on peut

(12) Et cela bien que le RD 1995/78, du 12 mai, qui contient le tableau en vigueur des maladies professionnelles, ne mentionne pas concrètement le stress, la dépression et autres maladies mentales, qui peuvent se produire suite au harcèlement moral. Récemment aboli par RD 1299/2006, du 10 novembre, par lequel on approuve le tableau de maladies professionnelles dans le système de la Sécurité sociale et on établit des critères pour leur notification et registre (BOE du 19 décembre de 2006), mais sans modification en ce sens. Cette carence légale est dépassée, généralement, en recourant à l'extension du concept d'accident du travail et en appliquant les dispositions de l'article 115 de la loi générale de Sécurité sociale, où on définit l'accident du travail comme «[...] toute lésion corporelle que le travailleur subit à l'occasion ou par conséquence du travail qu'il exécute pour le compte d'un autre [...]». La même solution juridique en droit francais, $c f$. BADEL 2006, pp. 918 et suivantes.

(13) $C f$., parmi de nombreuses décisions, STS du 15 janvier 2005 (RJ 1157); STSJ Madrid 17 mai 2004 (AS 2165); STSJ Andalucía/Granada du 29 avril 2003 (PROV 160104); STSJ Cataluña 15 mai 2003 (AS 2497); SSTSJ Navarra 30 avril 2001 (AS 1878), 18 mai 2001 (AS 1821) et 15 juin 2001 (PROV 230916); STSJ Castilla y León, du 20 décembre 2001 (AS 198).

(14) Pour une étude de son contenu, vid. Pérez Del Río, T.: «La protección frente a la discriminación en el Derecho comunitario: las D/2000/43/CE, D/2000/78/CE y D/2002/73/CE», Revista de Derecho Social, ${ }^{\circ}$ 19, 2002.

(15) Proposition de loi $\mathrm{n}^{\mathrm{O}} 122 / 000157$ dans laquelle on sollicitait l'inclusion du harcèlement moral au travail comme une infraction standardisée dans l'article 314 bis Code pénal, la modification du statut des travailleurs pour inclure la protection face à des conduites constitutives de harcèlement moral en tant que droit des travailleurs dans l'article 4.2, paragraphe e), dans l'article 17.1 comme cause de discrimination, dans l'article 50.1. c) comme cause pour la demande de résiliation du contrat de travail à la demande du travailleur touché par le harcèlement moral et dans l'article 54.2. c) comme cause de renvoi disciplinaire. néanmoins détacher certains éléments structurels sur lesquels il existe un certain consensus (16):

a) Violence ou maltraitance psychologique: on considérera comme telle une conduite ou une action de harcèlement, de persécution ou de violence psychologique contre une personne ou un ensemble de personnes, qui ne doit pas être confondue avec une simple situation de tension dans le travail ou de mauvais climat de travail, fréquente dans certaines entreprises ou certains établissements et parfois consubstantielle à certains environnements de travail problématiques (17).

b) Un comportement persistant, systématique et réitéré dans le temps qui doit donc être exercé de manière systématique et pendant un temps prolongé que, par exemple, la NTP 476 de l'INSH, déjà mentionnée, fixe à six mois. Cependant, ce délai ne doit pas être considéré comme une référence absolue puisque l'important n'est pas tant la durée, mais l'idée d'une continuité dans la violence visant à la destruction psychologique ou morale du travailleur. c) Avec l'intention de blesser personnellement la victime, c'est-à-dire, que doit clairement apparaître une intention de nuire psychiquement, professionnellement et socialement à la personne qui subit le harcèlement. On doit constater l'existence d'un plan spécifique de «poursuite», dans le but de déstabiliser et de miner émotionnellement la personne, en créant un environnement de travail humiliant et hostile, dans le but de détériorer sa capacité de travail et se débarrasser d'elle (18).

d) Il n'est pas nécessaire que la victime souffre de dommages quant à sa santé physique ou psychique, comme il semble ressortir de certaines décisions de justice examinées (SJS Madrid du 18 juin 2001 et de Murcie du 30 mai 2003).

e) L'existence d'un pouvoir asymétrique : il ne s'agit pas nécessairement d'un pouvoir hiérarchique; le harcèlement moral se produit entre des sujets inégaux, de sorte que le «harceleur» se trouve en position de supériorité par rapport au «harcelé» (19). On ne peut pas faire abstraction du fait que les relations de travail sont des relations hiérarchiques

(16) Cavas Martínez, 2005, pp. 5 et suivantes. Dans un sens identique, Molina Navarrete, 2001, pp. 1142 et suivantes; Agra Viforcos, Fernández Fernández, Tascón López, 2002, pp. 7 et suivantes; Sanahuja VIDal, 2002,pp. 642 et suivantes; Correa Carrasco, Valdés de la Vega (2007).

(17) Sur la distinction toujours délicate entre le harcèlement moral et les tensions ou les pressions dans le travail, voir les sentences de la Cour du secteur social de Murcie du 7 mars 2003 et celle du TSJ d'Andalousie de du 9 novembre 2005. Toutes les deux ont été commentées, la première par CORDERO SAAVEdRa, 2003 et la deuxième par BorRajo Dacruz, 2006.

(18) «[...] la volonté délibérée de réaliser une humiliation ou une vexation est un élément constitutif du comportement qui nous occupe, mais non nécessaire, au moins lorsqu'il s'agit d'évaluer les conséquences au plan professionnel d'un certain comportement de mobbing [...]», comme l'a indiqué FERNÁNDEZ LÓPEZ, 2002, p. 59.

(19) $C f$. entre autres, Pineda Segura, 2001, p. 146; Mella MÉNDEZ, 2003, p. 16. 
dans lesquelles, de manière inhérente, se manifeste l'existence d'intérêts opposés et qui, en tant que telles, impliquent que des personnes ordonnent et que d'autres obéissent (subordination/domination). Toutefois, le pouvoir qui permet au harceleur de développer son comportement de harcèlement ne doit pas nécessairement dériver d'une situation de hiérarchie par rapport au harcelé (relation verticale), il peut s'agir aussi bien d'une situation de supériorité de fait (horizontale) (20).

\section{Le rôle de la législation en matière de prévention des risques professionnels}

L'œuvre de l'Organisation internationale de travail (OIT) sur les questions de sécurité et de santé au travail a été d'une importance cruciale. Il existe plus de soixante-dix conventions et recommandations de l'OIT à ce sujet, accompagnées en outre d'une importante tâche de diffusion qui a donné lieu à la publication de plus de trente répertoires de recommandations pratiques sur la sécurité et la santé au travail. Parmi toutes ces normes internationales, c'est la convention $\mathrm{n}^{\circ} 155$ sur la sécurité et la santé des travailleurs de 1981 qui constitue le cadre de référence servant d'appui à une culture en matière de sécurité et de santé au travail. D'autre part, les lignes directrices de l'OIT relatives aux systèmes de gestion de la sécurité et la santé au travail (ILOOSH 2001) constituent un puissant instrument pour développer une culture viable de la sécurité et la santé dans les entreprises, ainsi que des mécanismes contribuant à améliorer l'environnement de travail.

De plus, la $91^{\mathrm{e}}$ réunion de la Conférence internationale du travail (2003) a affirmé que «une culture nationale de prévention en matière de sécurité et de santé au travail implique le respect du droit de jouir d'un environnement de travail sûr et sain à tous les niveaux, la participation active des gouvernements, des employeurs et des travailleurs pour assurer un environnement de travail sûr et sain à travers un système de droits, de responsabilités et de devoirs définis, et l'attribution de la priorité maximale au début de la prévention. Afin d'instaurer et de maintenir une culture de prévention en matière de sécurité et de santé, on doit employer tous les moyens disponibles pour augmenter la sensibilisation, la connaissance et la compréhension générale en ce qui concerne les concepts de danger et de risque, ainsi que la manière de les prévoir et de les contrôler».

Plus récemment, cette préoccupation a porté ses fruits, puisqu'au moment où nous écrivions

(20) Il s'ensuit que le harcèlement peut se présenter de manière verticale ou horizontale. Sur ce dernier aspect, $c f$. GALÁN VEGA, 2005. ces lignes, a été adoptée, dans le cadre de la $95^{\mathrm{e}}$ réunion de la Conférence internationale du travail (2006) (21), une nouvelle convention ( $\mathrm{n}^{\circ}$ 187/2006) sur un cadre promotionnel pour la sécurité et la santé au travail et une recommandation $\left(n^{\circ} 197 / 2006\right)$ sur le même sujet(22), qui sont basées sur la stratégie globale de l'OIT en matière de sécurité et de santé au travail adoptée au cours de la Conférence internationale de 2003. Ces normes insistent sur la nécessité de promouvoir le développement d'une «culture de sécurité» qui donne une plus grande importance au sujet de la sécurité et de la santé au travail dans les agendas nationaux.

Une étude de la réglementation internationale sur ce sujet ne peut manquer de se référer à la charte sociale européenne, traité qui est le fruit de l'action $\mathrm{du}$ Conseil de l'Europe, organisme international à l'origine du règlement des droits politiques et sociaux à l'échelle européenne. La version première de la charte sociale européenne date de 1961 et a été ratifiée par l'Espagne le 6 mai 1980. Elle contient des droits du travail «basiques», mais elle a été postérieurement modifiée à plusieurs reprises pour en étendre le contenu initial. Ces modifications ont donné lieu à la charte sociale européenne révisée qui est entrée en vigueur le $1^{\text {er }}$ juillet 1999 et qui n'a pas été ratifiée par l'Espagne. L'article 26 de la charte sociale européenne révisée contient le droit des travailleurs à la dignité, qui implique, entre autres, la promotion d'une prise de conscience et de la prévention du harcèlement au travail. Bien que cette disposition n'oblige pas les parties à promulguer une législation spéciale à ce sujet, elle invite à l'adoption de mesures adéquates pour protéger les travailleurs.

Quant à la réglementation communautaire en la matière, rappelons que la directive-cadre 89/391/ CEE constitue le cadre juridique général de la politique communautaire de prévention de risques professionnels. Elle établit des droits et des obligations de contenu prédéfini, au moyen de standards de régulation fermés. Le droit communautaire a cependant adopté un modèle de régulation complexe qui inclut une diversité de mécanismes, de procédures régulatrices et de techniques visant à garantir l'efficacité du droit. Le droit communautaire table sur un développement ouvert et flexible de règles qui tiennent compte de la diversité des situations, de l'évolution des organisations, des techniques productives et des instruments préventifs. On pourrait ici faire un compte rendu de l'adoption de diverses directives communautaires en matière de santé et de sécurité au travail, qui ont supposé la communautarisation d'aspects décisifs de la prévention de risques professionnels et qui ont provoqué une transformation profonde

(21) Ils peuvent être consultés sur le site www.ilo.org/public/ (22) $C f$. www.ilo.org/global/About_the_ILO 
des ordres juridiques nationaux en général et du système espagnol en particulier.

Toutefois et concrètement, en ce qui concerne la question qui nous occupe ici, c'est-à-dire le harcèlement moral au travail, et bien qu'ait été prévue la nécessité d'une étude par la Commission sur la pertinence et le cadre d'application d'un instrument communautaire, aucune des directives existantes ne se réfère spécifiquement aux risques psychosociaux et à leur prévention en tant risques professionnels. Le Parlement européen a cependant approuvé une résolution sur le harcèlement moral sur le lieu de travail (2001/2339 (INI) du 20 septembre), en vertu de laquelle la Commission est incitée à étudier la possibilité de clarifier ou d'étendre la directive-cadre sur la santé et la sécurité au travail, ou même d'élaborer une nouvelle directive cadre qui constituerait un instrument juridique pour combattre le harcèlement moral en tant que problématique spécifique relevant de la sécurité et de la santé au travail.

Du point de vue de la législation nationale, il est nécessaire de commencer par attirer l'attention sur le fait que la nécessité d'aborder juridiquement le harcèlement moral au travail dérive de la dimension constitutionnelle que présente le sujet, étant donné que le harcèlement moral suppose une atteinte à l'intégrité morale de la personne. En effet, on considère que «le harcèlement moral constitue une conduite complexe dans son contenu et dont les effets sont multiples du point de vue des atteintes qu'il comporte: il suppose toujours une atteinte à la dignité personnelle et à l'intégrité morale de la victime et, éventuellement, à d'autres droits constitutionnellement protégés » (SERRANO Olivares, 2005). L'attention est donc portée sur l'obligation des pouvoirs publics espagnols de promouvoir les conditions permettant que les droits et les libertés des personnes exprimés par l'article 9 de la Constitution espagnole (CE) soient réels et effectifs. En relation avec ce précepte, l'article $10 \mathrm{CE}$ établit que la dignité des personnes, les droits inaliénables qui leur sont propres, le libre développement de la personnalité et le respect des droits des autres sont le fondement de l'ordre juridique et de la paix sociale et l'article $15 \mathrm{CE}$ reconnaît le droit à la vie et à l'intégrité physique et morale. Il est donc tout à fait indiscutable que «la santé et la sécurité du travailleur est la matière dans laquelle la dimension constitutionnelle de l'implication de la personne dans le contrat de travail s'avère la plus évidente» (RODRÍGUEZ-PIÑERO, 2005).

Quant au développement légal des contenus constitutionnels, signalons l'article 4.2 du Statut des travailleurs (ET) qui inclut expressément dans la protection de la dignité de la personne - en tant que droit fondamental du travailleur-la protection face au harcèlement pour raisons d'origine ethnique, religieuse, de convictions, d'invalidité, d'âge ou d'orientation sexuelle. On peut constater que, malgré l'incorporation avec la loi no 3/2007 du 22 mars sur l'égalité effective entre les femmes et les hommes(23), d'une définition explicite sur le comportement générant des situations de harcèlement fondé sur le sexe, c'est une nouvelle occasion perdue de procéder à une incorporation explicite du harcèlement moral, car on s'est contenté de traiter uniquement du harcèlement discriminatoire (sexuel ou en raison du sexe), qui suppose des motifs discriminatoires. Plus précisément, il est considéré comme «toute conduite selon le sexe d'une personne ayant pour objet ou pour effet de porter atteinte à la dignité et de créer un environnement intimidant, humiliant ou offensant» (article 7.2.). Toutefois, le législateur a choisi pour ce type de comportement un traitement conjoint avec le harcèlement sexuel et le droit espagnol continue, avec cette option, sans une référence explicite au harcèlement moral. Malgré cela, et en vertu d'une interprétation extensive, on peut considérer, comme il a été fait jusqu'à présent, que la protection face à des situations de harcèlement moral au travail est comprise dans la garantie de la dignité de la personne.

Par ailleurs, dans l'article 50 ET apparaît une autre référence au droit à la dignité, dans la mesure où cette disposition établit les causes pour lesquelles le travailleur peut solliciter la résiliation du contrat de travail ou considérer comme constituant des modifications substantielles des conditions de travail les actes qui portent préjudice à sa formation professionnelle ou comportent une atteinte à sa dignité(24). L'article 54.2 c) ET considère aussi les conduites constitutives de harcèlement moral comme une cause de licenciement disciplinaire en ce qu'elles constituent des offenses physiques ou verbales.

Quant au traitement du harcèlement moral du point de vue de la réglementation de la prévention de risques professionnels(25), on indiquera tout d'abord que cette réglementation repose sur une prémisse de base qui est le devoir général de protection qui incombe au chef d'entreprise (article 14 de la loi de prévention des risques au travail, désormais

(23) Cette loi intègre les deux directives communautaires sur l'égalité de traitement, la directive 2002/73/CE, relative à l'application du principe de l'égalité de traitement entre hommes et femmes en ce qui concerne l'accès à l'emploi, la formation et la promotion des professionnels, et les conditions de travail; et la directive 2004/113/CE sur la mise en œuvre du principe de l'égalité de traitement entre hommes et femmes dans l'accès aux biens et services et des fournitures (PEREZ DEL RIO, 2002). (24) L'hypothèse classique considérée dans cet article et qui apparaît dans la doctrine jurisprudentielle est l'assignation au travailleur de fonctions de niveau et d'importance inférieurs par rapport à la formation, l'expérience et les fonctions du travailleur, sans justification, de manière permanente (STSJ la Galice 19 février 1996 et SSTS 18 septembre 1990 et 26 décembre 1990).

(25) Constituée par la loi 31/1995, de prévention de risques professionnels (LPRL) et le règlement 39/1997, de services de prévention (RSP). 
LPRL) (Alarcón CARACuel, 1996, pp. 107 et 108). La principale conséquence juridique ne peut en être que celle-ci: c'est le chef d'entreprise, en tant que responsable de l'organisation productive et débiteur de l'obligation de sécurité dans le cadre du contrat de travail, qui doit évaluer et prévoir tous les risques professionnels et, parmi ceux-ci, les risques psychosociaux. Cette obligation préventive étant conçue en des termes très larges, il importe peu que la réglementation espagnole fasse mention explicite des risques psychosociaux. Toutefois, certains ne manquent pas d'attirer l'attention sur le fait que, malgré «l'importance de ce type de comportements préventifs pour garantir efficacement le droit des travailleurs à une atmosphère de travail "saine", aucune modalité de harcèlement moral, ni même la modalité de harcèlement sexuel, n'est expressément mentionnée dans la LPRL» (Molina NaVARRETE, 2002). Cette première impression qui tient à une approche littérale de la règle ne doit cependant pas induire en erreur puisque la protection face à tout type de risques pour la santé, y compris les risques psychosociaux, est intégrée dans le cadre objectif et matériel d'application de la LPRL et dans le droit des travailleurs à une protection efficace en matière de santé au travail. C'est en lisant entre les lignes, tant de la LPRL que du RSP, qu'on peut trouver des références concrètes et opératoires, notamment dans des dispositions comme celles des articles $4.2,3$ et $7 \mathrm{~g}(26), 15.1 \mathrm{~d}$ et $\mathrm{g}(27)$ et 25 (28) de la LPRL et de 4(29) du RSP.

En définitive, le problème peut parfaitement être traité sur la base de la réglementation de la prévention de risques professionnels et des instruments de protection de la santé de travailleurs, malgré le défaut de références précises et spécifiques en matière de harcèlement moral. En effet, selon la conception préventive qui s'applique ici, il faut prendre en considération toute condition présente dans le travail susceptible d'être une source de risques pour l'intégrité, la vie ou la santé des travailleurs, afin d'en prévoir les possibles conséquences nuisibles. La LPRL impose au chef d'entreprise des devoirs généraux de prévention, avec des droits corrélatifs des travailleurs à un niveau adéquat de protection. Cela passe par l'organisation du milieu de travail, la mise en œuvre des moyens nécessaires et une tâche d'information et de formation des travailleurs, une amélioration des connaissances sur la prévention des risques et la promotion d'une attitude positive et d'une culture de

(26) Définition des concepts de risque professionnel, de dommages dérivés du travail et de conditions de travail.

(27) Parmi les principes généraux de l'action préventive, il faut souligner l'importance de la thématique de l'adaptation du travail à la personne et de la planification de l'action préventive.

(28) Il établit un règlement spécifique de protection des travailleurs qui se trouvent manifestement dans des états ou des situations transitoires qui ne répondent pas aux exigences psychophysiques des postes de travail respectifs.

(29) Paragraphe «contenu général de l'évaluation». prévention à tous les niveaux de l'entreprise avec un rôle significatif accordé aux interventions collectives dans la phase d'élaboration et d'application des politiques de prévention dans l'entreprise. En accord avec l'article 14 LPRL, le chef d'entreprise doit garantir la sécurité et la santé des travailleurs qui sont à son service, en adoptant dans le cadre de ses responsabilités toutes les mesures nécessaires à une telle fin, en appliquant un système de gestion et de planification des activités préventives et en se dotant d'une organisation et des moyens nécessaires. Sur la base de telles prévisions, on a la garantie que la législation spécifique englobe tous les risques qui peuvent avoir une origine professionnelle, ce qui inclut les risques psychosociaux.

Un point supplémentaire: la loi 54/2003 qui est venue réformer le cadre normatif de la prévention de risques professionnels n'a pas non plus incorporé de règles particulières en ce qui concerne les risques psychosociaux en général, ni le harcèlement moral en particulier, le législateur espagnol laissant passer une occasion dont ont su, au contraire, profiter d'autres pays proches. C'est le cas de la loi française de modernisation sociale (loi $\mathrm{n}^{\circ} 73$ du 17 janvier 2002, JO du 18 janvier), par laquelle le législateur français a profité de l'occasion pour incorporer la définition du harcèlement moral dans le Code du travail et dans le Code pénal(30).

\section{Tableau 1}

La nouvelle notion de harcèlement moral dans le Code du travail et dans le Code pénal français selon la loi de modernisation sociale du 17 janvier 2002

\begin{tabular}{|c|c|}
\hline Code du travail & Code pénal \\
\hline $\begin{array}{l}\text { Article L. } 122-49 \text { - «Aucun } \\
\text { salarié ne doit subir les agis- } \\
\text { sements répétés de harcèle- } \\
\text { ment moral qui ont pour objet } \\
\text { ou pour effet une dégradation } \\
\text { des conditions de travail } \\
\text { susceptible de porter atteinte } \\
\text { à ses droits et a sa dignité, } \\
\text { d'altérer sa santé physique ou } \\
\text { mentale ou de compromettre } \\
\text { son avenir professionnel». }\end{array}$ & $\begin{array}{l}\text { Article 222-33-2 - «Le fait } \\
\text { de harceler autrui par des } \\
\text { agissement répétés ayant } \\
\text { pour objet ou pour effet une } \\
\text { dégradation des conditions } \\
\text { de travail susceptible de } \\
\text { porter atteinte à ses droits } \\
\text { et à sa dignité, d'altérer sa } \\
\text { santé physique ou mentale ou } \\
\text { de compromettre son avenir } \\
\text { professionnel, est puni d'un } \\
\text { an d'emprisonnement et de } \\
15000 \text { euros d'amende». }\end{array}$ \\
\hline $\begin{array}{l}\text { Article L. 152-1-1 - «En cas } \\
\text { de violation de l'article L. 122- } \\
49 \text { est prévue une peine d'em- } \\
\text { prisonnement d'un an et une } \\
\text { amende de } 3750 \text { euros ». }\end{array}$ & \\
\hline
\end{tabular}

(30) Pour une étude exhaustive desdits préceptes et de la loi elle-même, $c f$. entre autres, Mazeaud, 2002, pp. 321 et suivantes; Delga, RaJkumar, 2005, pp. 161 et suivantes; LEROUGE, 2005 ; RADÉ, 2006, pp. 826 et suivantes ; RADÉ, 2006 , Cahier Juridique RF Social, pp. 58 et suivantes. 
Toutefois, la loi espagnole déjà citée a contribué à renforcer le devoir général de prévention du chef d'entreprise, en modifiant les articles 14 et 16 de la LPRL qui indiquent que l'obligation patronale en matière de prévention des risques professionnels procède par l'intégration de l'activité préventive dans le système général de gestion de l'entreprise.

\section{Le rôle de la tutelle préventive dans la LPRL espagnole}

À partir du moment où les risques psychosociaux sont reconnus comme des risques professionnels, la conséquence est évidente: on doit appliquer à ce type de situations toutes les normes générales relatives à la gestion des risques professionnels, c'està-dire l'obligation d'identification et d'élimination des risques, ainsi que l'évaluation de ceux qui n'ont pas pu être évités, en appliquant les mesures résultant de l'évaluation par le biais d'une action prévue pour la prévention de ces risques (articles 3-9 et 15-16 LPRL), l'obligation de donner une formation à tous les travailleurs pour ce qui est des risques propres à leur poste de travail (article 19 LPRL), l'obligation d'adapter les conditions du poste de travail aux conditions personnelles et à l'état biologique du travailleur (articles 15.1. d et 25 LPRL), celle de mener à bien les activités de prévention par le recours à des experts justifiant de la formation adéquate et dont la mission peut être confiée à des travailleurs désignés par l'entreprise ou qui peuvent s'intégrer dans un service de prévention propre ou externe, dans lequel il devra y avoir des experts dans l'évaluation et le diagnostic de risques psychosociaux (articles 30 et 31 LPRL), l'obligation de surveillance de la santé pour la détection de maladies psychiques (article 29 LPRL), les droits de participation et d'information des travailleurs et de leurs représentants en matière de prévention des risques professionnels (articles 18 et 33 à 40 LPRL), ainsi que les devoirs de coopération et de coordination des chefs d'entreprise qui se trouvent dans un même lieu de travail (article 24 LPRL).

Cependant, les principes généraux de l'action préventive et, plus concrètement l'évaluation et la planification, ne peuvent pas être appliqués d'une manière mécanique en ce qui concerne les risques psychosociaux. Et cela parce que l'activité préventive dans ce cadre requiert d'affronter le harcèlement moral sur le lieu de travail, ce qui suppose un engagement sérieux de toute la ligne hiérarchique de l'organisation (CAVAS MARTínez, 2005). Si, comme on l'a déjà signalé, c'est le chef d'entreprise qui est obligé de prévoir l'apparition de possibles comportements de harcèlement moral dans l'entreprise, il est évident qu'il devra pour cela adopter les mesures préventives correspondantes. Dans les cas où les mesures préventives s'avéreraient inutiles mais où ce type de risque se produirait finalement, le chef d'entreprise devra adopter des mesures efficaces et immédiates pour y remédier et éviter que cela ne se reproduise. C'est la conséquence de l'obligation de sécurité du chef d'entreprise envers ses employés, qui implique qu'il n'y ait pas de harcèlement du chef envers les employés ni d'un employé envers un autre, obligation dont il doit assurer l'efficacité.

Autrement dit, la conception et la mise en pratique de cette politique préventive par rapport au risque de harcèlement moral sur le lieu de travail est un problème technique, qui doit être traité selon les modalités d'organisation technique de la prévention établies dans chaque entreprise, selon les options ouvertes par l'article 30 LPRL. L'évaluation tant des conditions objectives qui affectent le poste de travail (facteurs environnementaux et organisationnels) que des données subjectives se rapportant au travailleur (sensibilité particulière à certains risques), bien qu'elle puisse s'appuyer sur des codes ou des guides «normalisés» conformément aux expériences connues, exigera une capacité de compréhension particulière, de la part du technicien spécialisé en ergonomie et en psychologie, appliquée à la prévention. Ces situations sont de celles qui requièrent non seulement l'établissement d'une stratégie de mesure spécifique pour s'assurer de la fiabilité des résultats, mais aussi et surtout une interprétation ou une approche circonstanciée des données car, à défaut de critères d'évaluation, il faudra s'en remettre principalement à l'appréciation professionnelle et accréditée d'un technicien de niveau supérieur et aux méthodes ou aux critères figurant dans les guides de l'INSHT (tels que NTP 476/1998).

Dans ce cadre de la prévention, la tâche des techniciens de prévention prend une importance particulière, ainsi que l'intervention des représentants des travailleurs ayant des compétences en matière de prévention des risques professionnels (délégués du personnel et comités d'entreprise, délégués de prévention et comités de sécurité et de santé). Et cela du fait que «le problème préventif que suscite le risque de harcèlement moral n'est pas seulement une question technique, mais aussi et surtout un problème politique, c'est-à-dire de politique d'entreprise. Par conséquent et conformément au principe de santé intégrée dans la gestion d'entreprise, il requiert l'adoption d'une vaste gamme de mesures organisationnelles qui sont définies par l'entreprise et qu'il convient d'appliquer effectivement à toute personne qui a une responsabilité dans le processus de prise de décisions en ce qui concerne la gestion du personnel (Molina Navarrete, 2001). Parmi les différentes mesures à adopter dans le processus de planification et d'intervention, les principales sont les suivantes:

- mesures d'organisation, puisque le problème du harcèlement moral est lié dans un très grand nombre de cas à des insuffisances dans l'organisation du travail; 
- mesures de type éducatif ou culturel, visant à prévoir de manière générale et anticipée ces comportements ou à éviter qu'ils soient réitérés ;

- mesures de procédure de résolution des conflits interpersonnels, comme la reconnaissance de procédures simples, impartiales et claires permettant de formuler des plaintes ou des réclamations, en vue de protéger efficacement la position professionnelle des intéressés, etc.;

- et mesures à caractère d'aide, parmi lesquelles on inclut toutes les actions visant à fournir des traitements psychologiques et de réadaptation.

Sur la base de ce qui précède, l'entreprise doit adopter l'action préventive suivante, conformément à ce qui est prévu dans l'article 16 LPRL:

1) Dans l'évaluation initiale qui est déterminante pour l'identification adéquate des risques en vue d'élaborer la stratégie préventive la plus efficace, on pourra utiliser les méthodes ou les critères repris dans les normes UNE, les guides de l'Institut national de sécurité et d'hygiène au travail, etc., en l'absence de réglementation spécifique, en incluant l'analyse des risques psychosociaux, spécialement des aspects ou des facteurs tels que la surcharge de travail, les aspects organisationnels, la distribution de responsabilités, la prise de décisions, ainsi que les caractéristiques personnelles et psychologiques des travailleurs. Comme exemple, les protocoles de l'INSHT qui ont pris comme modèle le système scandinave en fixant une évaluation complète de risques psychosociaux. Ce modèle, contrairement à d'autres, utilise un système généraliste d'évaluation qui cherche à examiner les facteurs organisationnels qui peuvent influencer la santé mentale, la dignité des travailleurs et vise à établir des mesures pour empêcher la génération de risques psychosociaux. Ce dernier système présente l'avantage sur d'autres, comme les systèmes français ou belge plus centrés sur la gestion du conflit, ou sur les systèmes de conciliation et de renforcement des flux de communication dans les entreprises, de considérer aussi bien les atteintes contre la dignité comme le harcèlement, le stress professionnel et les autres risques psychosociaux. Cette évaluation devra être révisée et mise à jour quand auront changé les facteurs ou les conditions précédemment analysés (changements organisationnels, nouvelles technologies, restructuration du personnel, manque de ressource, charge de travail, changements dans les conditions personnelles ou biologiques d'un travailleur), quand se produira une certaine hypothèse de harcèlement et quand, de cette hypothèse, en résulteront certains dommages pour la santé des travailleurs. Une fois le risque apparu, il va s'avérer obligatoire d'évaluer dans quelle mesure il peut dégénérer, afin d'adopter les mesures opportunes pour le combattre et de prévoir de nouvelles manifestations du harcèlement dans le futur.

2) Établir un plan de prévention dans lequel on intègre des mesures préventives visant à éviter les conflits de relations et le harcèlement moral ou, si celui-ci apparaît, à éviter les répercussions sur la santé des travailleurs; à mettre en place la formation, l'information et une surveillance périodique de la santé. Le plan de prévention des risques professionnels devra inclure la structure organisationnelle, les responsabilités, les fonctions, les pratiques, les procédures, les processus et les ressources nécessaires pour effectuer l'action de prévention de risques dans l'entreprise. En ce sens, la note technique de prévention 476 de l'INSHT à laquelle on a déjà fait référence affirme que "le substrat qui favorise l'apparition de ce type de conduites est lié à deux aspects: l'organisation du travail et la gestion des conflits par les supérieurs» et ces deux aspects se trouvent privilégiés dans le plan de prévention.

Cependant, la situation espagnole en ce qui concerne l'évaluation des risques psychosociaux en est encore aux prémisses pour des raisons diverses, parmi lesquelles on mentionnera le manque de méthodologie adéquate et la difficulté qu'une telle tâche constitue (Schaufeli, Salanova, 2002, pp. 4 et suivantes).

\section{* \\ * *}

La répercussion sociale que le harcèlement moral au travail paraît avoir connue dans la période récente $\mathrm{a}$ eu, toutefois, une incidence très faible du point de vue de la structure institutionnelle et juridique des relations de travail en Espagne, plus faible en tout cas que dans les pays voisins avec lesquels la situation espagnole peut être comparée. Comme on l'a mis en évidence, cela n'a pas donné lieu à une réglementation spécifique, ni à une ratification des traités internationaux qui contiennent une définition juridique de cette notion. Dans ce sens, on peut affirmer que dans l'ordre juridique espagnol, le traitement juridique du harcèlement moral au travail est encore tributaire des obligations contractées par l'État espagnol quant à l'application de la réglementation internationale et communautaire; il est par conséquent déficitaire et insuffisant (Romero RóDENAs, 2005 , pp. 19 et suivantes). Si à tout cela on ajoute le fait avéré que la jurisprudence espagnole a été erratique et peu claire sur le sujet, le panorama qui en résulte est désolant.

Que l'ordre juridique espagnol en général et le droit du travail en particulier, n'aient pas été capables d'aller au-delà de la perspective réparatrice classique peut paraître surprenant et devrait prêter à réflexion. En ce sens, il est absolument nécessaire de développer et d'appliquer de nouvelles politiques de prévention des risques professionnels à la lumière des décisions de justice mettant en pratique la législation espagnole et de l'évolution préoccupante des dommages causés dans le cadre professionnel. Et cela parce que c'est déjà un lieu commun 
de considérer qu'il y a eu une incorporation déficiente de ce nouveau modèle de prévention des risques professionnels de la part de la prétendue loi de prévention de risques professionnels, tant dans la version initiale de 1995 que dans sa réforme de 2003, principalement en raison du fait d'une application plus formelle qu'efficace de la réglementation et par manque d'adéquation de celle-ci aux nouvelles formes d'organisation du travail (31).

Probablement tout cela est-il dû au fait que le succès de cette norme "dépendait et dépend non seulement de l'établissement et du respect des règles, même si celles-ci et leur observance sont encore des éléments indispensables pour garantir la sécurité et la santé au travail, mais aussi de l'instauration de compromis, d'attitudes et de motivations en créant une sensibilité et une culture environnementale et de sécurité dans l'entreprise, à laquelle la loi n'est pas suffisamment parvenue» (RoDRÍGUEZ PIÑERo, 2005). De plus, devant l'alourdissement des exigences légales et des contrôles de l'administration publique, la pratique patronale a dérivé vers une application rigoureuse des exigences documentaires afin de satisfaire au contrôle externe et formaliste des instances publiques et d'éviter les sanctions administratives. Cette «portée prophylactique» (CRUZ VILLALÓn, 2006) des normes de sanction et de l'imputation de responsabilités économiques n'est pas sans mérites, d'un point de vue juridique positiviste, mais ce jugement doit être nuancé dans la mesure où cela se produit au détriment de l'efficacité pratique à laquelle prétendent de telles mesures.

Dans un contexte tel que celui qui vient d'être exposé, l'idéal serait que les nouvelles techniques soient conçues comme complémentaires et susceptibles d'enrichir le cadre institutionnel déjà existant. En même temps, se manifeste la nécessité d'incorporer de nouvelles techniques de protection plus adaptées aux nouvelles exigences de flexibilité des entreprises, dérivées d'une économie ouverte. Autrement dit, il s'agit de transiter d'un modèle dans lequel on s'est focalisé sur la fixation de devoirs et de responsabilités dont l'inaccomplissement entraîne des sanctions économiques, à un autre modèle qui ne doit pas chasser l'ancien mais dans lequel l'accent doit être mis sur l'élément «organisationnel», afin de mettre véritablement en marche des mécanismes de prévention et, de cette façon, étendre la «culture de la prévention».

Dans une perspective strictement préventive, c'est le chef d'entreprise, comme il a été dit à plusieurs reprises, qui est tenu d'évaluer et de faire face aux risques psychosociaux qui présentent une relation

(31) Comme on peut le lire dans l'exposé des motifs de la loi 54/2003 qui a tenté de compléter cette approche excessivement répressive de la réglementation de prévention de risques professionnels dans sa version originale, c'est-à-dire, dans la loi 31/1995. directe de cause à effet avec le travail, afin d'éviter des comportements dans le cadre de la relation de travail attentatoires à la dignité des personnes (à la sienne propre et à celle de ses employés). N'oublions pas que, dans le cadre juridique-préventif existant, il est parfaitement possible de contrôler le défaut d'évaluation des risques psychosociaux, ainsi que l'absence ou l'insuffisance de mesures préventives, c'est-à-dire de contrôler le respect de la double obligation qui pèse sur les employeurs afin de garantir une protection efficace des travailleurs. La ligne à suivre doit, par conséquent, être fondamentalement préventive tout en se situant sous l'empire de cette nouvelle approche de la sécurité et de la santé au travail introduite par la LPRL qui, au-delà de la simple application de mesures de sécurité de caractère technique, se traduit par l'intégration nécessaire de l'activité préventive dans toutes les décisions et actions patronales dans l'entreprise, dans tout cadre et à tous les niveaux hiérarchiques de cette dernière.

En tout état de cause, on ne saurait terminer cette analyse sans relever la nécessité d'une législation spécifique qui contribue à clarifier et à résoudre les nombreux problèmes qui demeurent sans réponse légale, ce qui est source d'une grande insécurité juridique dans le domaine abordé ici, qui revêt une grande importance sociale. Dans ce contexte, les travailleurs sont dans un certain sens désemparés face à la problématique du harcèlement dans le cadre du travail, et ils en sont réduits à exercer des recours dans la plupart des cas fondés sur le droit civil de la réparation des dommages non spécifiques. Tout cela se produit avec, comme conséquence, une dégradation des conditions de travail. Or, à défaut de législation spécifique, et en dépit des possibilités générales d'action ouvertes par le système espagnol de prévention de risques professionnels pour éviter ou corriger ces comportements qui nuisent aux conditions de travail, apparaît clairement l'exigence d'un engagement de la part des directions d'entreprises et des interlocuteurs sociaux. Autrement dit, il s'agit de créer une organisation du travail claire et transparente, qui évite ce que l'on appelle les «organisations toxiques » (Cubo Mayo, Pérez-Bustamante MOURIER, 2006) L'adoption de ces mesures requiert des actions de formation des travailleurs et de leurs représentants et des cadres moyens, des actions de coordination entre les entreprises et, en définitive, l'engagement du chef d'entreprise et de la direction du personnel dans une politique préventive et, en général, d'une politique de lutte contre la violence psychologique dans le milieu de travail. Cette politique doit être promue par les interlocuteurs sociaux et elle suppose l'introduction de clauses antimobbing dans les conventions collectives; elle exige aussi de la part des structures représentatives actuelles qu'elles prennent ce problème au sérieux et agissent en conséquence.

Comme l'a écrit le Conseil économique et social français, «par-delà le coût humain, déjà significatif 
et a lui seul difficilement tolérable, les pathologies et autres troubles psychologiques peuvent induire pour les entreprises des conséquences, tant du point de vue de leur performance et de leur compétitivité qu'en termes de dépenses de santé. La préservation de la santé au travail dans toutes ses dimensions, y compris la santé mentale, doit de ce fait représenter un enjeu sociétal pour l'ensemble des acteurs, dans une vision plus globale de santé publique (32)».

(32) Conseil économique et social (CES), Rapport sur les nouveaux risques pour la santé des salariés, 2004, CES, p. I11, sur le site http: //www.ces.fr/ces_dat2/2-3based/frame_ rech avis.html.

\section{Bibliographie}

Agra Viforcos B., Fernández Fernández R., Tascón LÓPEz R. (2002), «El acoso moral en el trabajo (mobbing): análisis sobre la limitada respuesta jurídica a un antiguo problema de reciente estudio y futura solución legal», Estudios financieros, Revista de Trabajo y Seguridad Social, no pp. 233-234.

Agence EUROPÉENNE pour la SÉcurité et la santé au TRAVAIL (2002), «Stress au travail», Facts, http ://ew2002. osha.europa.eu/

Alarcón Caracuel M.R. (1996), «Los deberes del empresario respecto a la seguridad y salud de sus trabajadores», dans Ojeda Avilés, A., Alarcón Caracuel, M.R. y Rodríguez Ramos, Ma. J. (coord.) (1996): La Prevención de Riesgos Laborales. Aspectos clave de la Ley 31/1995, Aranzadi, pp. 107 et 108.

BADEL M. (2006), «La souffrance au travail et le risque professionnel: la difficile appropriation du mal-être du salarié par le droit de la sécurité sociale», Revue de Droit sanitaire et social, $\mathrm{n}^{\mathrm{0}} 5$.

BONNECHÈRE M. (1994), «Le corps laborieux: réflexion sur la place du corps humain dans le contrat de travail», Droit Ouvrier, mai, pp. 173 et sqq.

Borrajo DACRUZ E. (2006), «Acoso moral y presión laboral», $A L, \mathrm{n}^{\circ} 3$.

Cavas Martínez F. (2005), «Derecho de la prevención y nuevos riesgos laborales», $A S, \mathrm{n}^{\circ} 18$.

Cisneros I y II (2001-2002), La incidencia del mobbing o acoso psicológico en el trabajo en España, Universidad de Alcalá de Henares, Informe.

Commission des Communautés européennes (2007), "Améliorer la qualité et la productivité du travail: une stratégie communautaire pour la santé et la sécurité au travail» (2007-2012), communication du 21 février 2007.
Cordero SAavedra L. (2003), «La delimitación jurídica entre el acoso moral y las tensiones laborales», $A S, \mathrm{n}^{\circ} 6$.

Correa Carrasco M., Valdés de la Vega B. (coord.) (2007), Los medios de tutela frente al acoso moral en el trabajo, Comares.

Cubo Mayo, A. y Pérez-Bustamante Mourier, J.P. (2006): «El derecho a la dignidad en el ámbito laboral. Acoso en el trabajo: moral y sexual...», $R L, \mathrm{n}^{\circ} 8$.

CRUZ Villalón J. (2006), «Ambiente de trabajo y nuevas técnicas normativas $», R L, \mathrm{n}^{\circ} 10$.

DASSA S. (2002): «Les nouveaux modes d'organisation du travail comme sources de problèmes de santé», Droit ouvrier, juin.

Delga J., Rajkumar A. (2005), «Le harcèlement moral: éléments caractéristiques du harcèlement moral au regard du Code du travail et de la jurisprudence contemporaine», Le Droit ouvrier, avril, pp. 161 et suiv.

FernÁndez López Ma . F. (2002), «El acoso moral en el trabajo ¿una nueva forma de discriminación?», Revista de Derecho Social, $\mathrm{n}^{\circ} 19$.

FONDATION EUROPÉENNE POUR L'AMÉLIORATION DES CONDITIONS DE VIE ET DE TRAVAIL (2007), Quatrième enquête pour l'amélioration des conditions de vie et de travail, sur le site http ://www.eurofound.europa.eu/

GALÁN VeGA Ma $M^{\mathrm{a}}$ L. (2005), «Acoso moral: mobbing horizontal», $A S, \mathrm{n}^{\circ} 17$.

García-Perrote Escarpín I., Mercader Uguina J.R. (coord.) (2007), La Ley de igualdad: consecuencias prácticas en las relaciones laborales y en la empresa, Lex Nova.

GonzÁlez de Rivera, J.L., Revuelta J.L. (2002), El maltrato psicológico. Cómo defenderse del mobbing y de otras formas de acoso, Espasa Calpe. 
HiRigoyen M.F. (1998), Le harcèlement moral. La violence perverse au quotidien, Syros.

Hirigoyen M.F. (2004), Malaise dans le travail. Harcèlement moral: Démêler le vrai du faux, La Découverte.

LAPEYRIÈRE, S. (2004), «Le harcèlement moral. Une affaire collective et culturelle», Travail et Emploi, $\mathrm{n}^{\circ}$ 97, janvier, pp. 29 et suiv.

Lerouge L. (2005), La reconnaissance d'un droit à la protection de la santé mentale au travail, LGDJ, DL.

Leymann H. (1996), Mobbing: la persécution au travail, Le Seuil.

Martín HernÁndez, Ma . L. (2005), «El deber del empresario de prevenir todos aquellos riesgos laborales que puedan llegar a producir algún tipo de daño psicológico sobre la salud de sus trabajadores », $A S, \mathrm{n}^{\circ} 16$.

Mazeaud A. (2002), «Harcèlement entre salariés : apport de la loi de modernisation», Droit social, no 3, pp. 321 et suiv.

Mella Méndez L. (2003), «El acoso psicológico en el derecho del trabajo», TS, $\mathrm{n}^{\circ} 145$.

Molina Navarrete C. (2001), «La tutela frente a la 'violencia moral'en los lugares de trabajo: entre prevención e indemnización», $A S$, Tomo $\mathrm{V}$.

Molina Navarrete, C. (2002), «Violencia moral en el trabajo: conducta prohibida y formas de tutela en los Derechos Europeos », Proceedings of the Second International Conference ORP 2002 on occupational risk prevention, sur le site www.prevencionintegral.com/

Morán Astorga C. (2002), El mobbing: persecución o psicoterror en el trabajo, Capital Humano, Cisspraxis.

OIT (2006), convention $\left(\mathrm{m}^{\circ} 187 / 2006\right)$ et recommandation (n ${ }^{\circ}$ 197/2006) sur un cadre promotionnel pour la sécu- rité et la santé au travail, sur le site http ://www.ilo.org/ global/About_the_ILO/Mainpillars/Therightsatwork/ Labour_Standards/

Pérez del Río T. (2002), « La protección frente a la discriminación en el Derecho comunitario : las D/2000/43/CE, D/2000/78/CE y D/2002/73/CE », Revista de Derecho Social, no 19.

Pineda Segura C. (2001), «El hostigamiento psicológico en el trabajo. El 'mobbing'», Estudios financieros, Revista de Trabajo y Seguridad Social, no 215.

PIÑUel I. (2001), Mobbing: cómo sobrevivir al psicoterror laboral, Sal Terrae.

RADÉ Ch. (2006), «Harcèlement moral et responsabilités au sein de l'entreprise : l'obscur éclaircissement», Droit social, $\mathrm{n}^{\mathrm{o}} 9-10$, pp. 826 et suiv.; Cahier juridique RF social, $\mathrm{n}^{\circ} 56$, pp. 58 et suiv.

RANDSTAD (2003), Calidad del trabajo en la Europa de los quince: el acoso moral, Informe IEL, ESADE-RANDSTAD, sur le site www.randstad.es/res/randstad/publicaciones/

Rodríguez PiÑERo M. (2005), «Nuevos enfoques de la prevención de los riesgos laborales», $R L$, tomo I.

Romero Ródenas Mª. J. (2004), Protección frente al acoso en el trabajo, Bomarzo.

Schaufeli W., Salanova Ma . L (2002), « ¿Cómo evaluar los riesgos psicosociales en el trabajo?», Revista seguridad y salud en el trabajo (INSHT), n 20, pp. 4 y ss.

Sanahuja Vidal M (2002), «Acoso moral en el trabajo: tratamiento jurídico (mobbing)», $A L, \mathrm{n}^{\circ} 30$, pp. 642 et suiv.

Serrano Olivares R. (2005), El acoso moral en el trabajo, CES.

ZWEIG S. (1997), Conscience contre violence ou Castellion contre Calvin, le Castor Astral. 